\title{
Year in Review 2014: Patient Safety
}

\author{
Dean R Hess PhD RRT FAARC
}

\author{
Introduction \\ Pressure Injury \\ Handoffs \\ Protocols and Multidisciplinary Teams \\ Perioperative Obstructive Sleep Apnea \\ Readmissions \\ Summary
}

\begin{abstract}
The topic of patient safety has received much attention in recent years. There are a number of areas in which the subject of patient safety impacts respiratory care practice. This paper focuses on articles published in $\mathbf{2 0 1 4}$ related to pressure injury, handoffs, protocols and multidisciplinary teams, perioperative obstructive sleep apnea, and readmissions. Key words: handoffs; patient safety; perioperative obstructive sleep apnea; pressure injury; protocols; readmissions. [Respir Care 2015;60(8):1197-1202. (C 2015 Daedalus Enterprises]
\end{abstract}

\section{Introduction}

The findings of the 2000 publication of "To err is human: building a safer health system" 1 was a surprise for many regarding hospital safety. A second report, "Crossing the quality chasm: a new health system for the 21 st century," 2 focuses more broadly on how the health-care delivery system can be designed to innovate and improve care. These reports together began a movement to improve

Dr Hess is affiliated with Respiratory Care Services, Massachusetts General Hospital, and Harvard Medical School, Boston, Massachusetts.

Dr Hess presented a version of this paper at the Year in Review 2014 of the AARC Congress 2014, held December 9-12, 2014, in Las Vegas, Nevada.

Dean Hess discloses relationships with Philips Respironics, PARI, Merck, Bayer, McGraw-Hill, Jones \& Bartlett Learning, UpToDate, and the American Board of Internal Medicine.

Correspondence: Dean Hess PhD RRT FAARC, Respiratory Care Services, Ellison 401, Massachusetts General Hospital, 55 Fruit Street, Boston, MA 02114. E-mail: dhess@ partners.org.

DOI: $10.4187 /$ respcare.04235 safety for hospitalized patients. This has evolved to become known as a patient safety culture, which is a form of organizational culture that includes the shared beliefs, attitudes, values, norms, and behavioral characteristics of employees. ${ }^{3,4}$ This then has the effect of influencing staff members' attitudes and behaviors in relation to their organization's patient safety performance. Unfortunately, a recent systematic review concluded that, despite strong face validity for a variety of patient safety culture strategies, there is limited evidence to support definitive impacts on patient safety climate outcomes..$^{5}$ Another systematic review reported evidence of relationships between patient safety culture and patient outcomes existing at the hospital and nursing unit level of analysis, but the number of studies finding statistically significant correlations is limited. ${ }^{6}$

In this review, I focus on specific strategies in which there is potential to positively affect patient safety related to respiratory care practice. This approach, focusing on specific practices, is different than an approach that evaluates a broader organizational culture of safety. I specifically address important papers published in 2014 dealing with pressure injury, handoffs, protocols and multidisciplinary care, perioperative obstructive sleep apnea (OSA), and readmissions. 


\section{Pressure Injury}

Nearly 10 years ago, the Centers for Medicare \& Medicaid Services (CMS) decided to disallow incremental payments associated with 8 secondary conditions that it deemed as preventable complications of medical care. ${ }^{7}$ If not present at the time of admission, these conditions are no longer taken into account in calculating payments to hospitals after October 1, 2008. Pressure ulcers are one of the complications on this list, and they accounted for 322,946 Medicare cases in the fiscal year 2006, with an average Medicare payment of $\$ 40,381$ for admissions in which the condition was present. For respiratory care practice, pressure ulcers can be related to the interface for noninvasive ventilation (NIV) and securing of artificial airways.

The role of NIV in appropriately selected patients is well established. ${ }^{8}$ Facial skin breakdown is a recognized complication of NIV, ${ }^{9}$ and the most common sites of skin damage during NIV are the bridge of the nose and the upper lip with a nasal mask, the nasal mucosa with pillows, and the axillae with a helmet. ${ }^{10}$ Skin ulceration can reduce comfort during NIV and contributes to intolerance of the interface and the success rate of NIV.

The objective of Barros et al ${ }^{11}$ was to study the effects of an oronasal interface using a 3-dimensional computational model with the ability to simulate and evaluate the main pressure zones of the interface on the face. The model simulated soft tissues, skull, and nasal cartilage. A computer simulation was performed to determine the pressure required to create the facial pressure zones. Consistent with clinical experience, the computer model identified several high-impact pressure zones in the nasal bridge and paranasal regions. This provides support for selection of an interface that best minimizes the risk of skin breakdown. Interfaces meeting this criterion include the total face and hybrid masks. Attention to how tightly the mask is secured, use of hydrocolloid dressings, and adjustment of the mask to reduce pressure on the face can also address this problem.

Yamaguti et $\mathrm{al}^{12}$ determined the frequency of skin breakdown and identified potential treatment-related risk factors for its development in adults with acute respiratory failure on NIV or CPAP. Subjects were retrospectively enrolled if they used NIV or CPAP for a period of $>2 \mathrm{~h}$. A stepwise forward logistic regression model was used to identify independent risk factors for skin breakdown. There were 375 subjects enrolled, and 14\% developed skin breakdown. The number of applications of NIV or CPAP for $>2 \mathrm{~h}$ was higher in subjects with skin breakdown than in those without. Subjects with skin breakdown also had a longer total duration of NIV use compared with those without skin breakdown. Most interestingly, subjects who developed skin breakdown had a greater use of the oronasal mask (93\%) compared with the group that used a total face mask (21.5\%). The adjusted odds ratio (OR) for skin breakdown was 80.8 for oronasal mask use (compared with total face mask use) and 8.6 for NIV or CPAP for $>26 \mathrm{~h}$. These results should help to inform the use of NIV in a way to reduce facial skin breakdown-in particular, use of a total face mask.

Many methods are available to secure the endotracheal tube, including tape and commercial devices. How the airway is secured has the potential to cause pressure ulcers. Fisher et $\mathrm{al}^{13}$ evaluated 16 endotracheal tube holders under a series of simulated clinical conditions. Measuring the distance displaced after a tug tested tube security. In addition, 9 of the 16 methods could be evaluated for speed of moving the tube to the opposite side of the mouth. Sensors located on a mannequin measured applied forces when the head was rotated vertically or horizontally. Median displacement of the tube by the tug test was $0 \mathrm{~cm}$, and the mean time to move the tube from one side of the mouth to the other ranged from 1.25 to $34.4 \mathrm{~s}$. Forces applied to the face with a vertical head lift ranged from $<0.2$ to $3.5 \mathrm{~N}$. Forces applied to the face with a horizontal rotation ranged from $<0.2$ to $3.52 \mathrm{~N}$. As a group, commercial devices produced greater force than noncommercial devices. The authors concluded that noncommercial airway holders exert less force on a patient's face than commercial devices. Many commercial holders allow for rapid but secure movement of the artificial airway from one side of the mouth to the other.

Wagner et al ${ }^{14}$ compared 7 different endotracheal tuberestraint combinations against themselves and one another at a series of test points covering a hemisphere on the plane of the face. Force values for tube motion of $2 \mathrm{~cm}$, $5 \mathrm{~cm}$, or failure were recorded for 3 pull tests, at each angle, for each method of tube fixation. All methods showed variation in the force required for tube motion with angle of force application. When forces were averaged over all test points for each fixation technique, differences as large as $132 \mathrm{~N}$ were observed. Compared with traditional methods of fixation (eg, tape), only one of the 3 commercially available devices consistently required a higher average force to displace the tube by 2 and $5 \mathrm{~cm}$. When ranges of force values for $5-\mathrm{cm}$ displacement were compared, devices spanned from 80 to $290 \mathrm{~N}$, whereas traditional methods spanned from 62 to $178 \mathrm{~N}$. Some methods were more reproducible than the others. The authors concluded that the efficacies of different fixation techniques are sensitive to the angle of force application. Traditional methods of tube fixation required similar extubation forces as devices commercially available in the United States. Unlike the study by Fisher et al, ${ }^{13}$ Wagner et al ${ }^{14}$ did not measure the force applied to the face.

Although rarely reported, tracheostomy wounds are commonly encountered in children. Use of maltodextrin gel and a silver alginate sponge might expedite healing of 
tracheostomy wounds. Hartzell et al ${ }^{15}$ conducted an 11month retrospective review of 11 children with wound complications following tracheostomy placement at a tertiary care facility. Wounds were in the infrastomal location $(n=8)$, stoma $(n=2)$, and lateral neck $(n=1)$. Wounds were stage 2 in 6 subjects, stage 3 in 4 subjects, and stage 4 in one subject. The authors reported that following treatment with maltodextrin gel and/or silver alginate, all wounds achieved complete epithelialization. The length of treatment ranged from 6 to $28 \mathrm{~d}$. No adverse effects related to maltodextrin gel or silver alginate were identified. Use of maltodextrin and/or silver alginate should be considered for the treatment of tracheostomy wounds in children.

\section{Handoffs}

Patient handoffs are a key source of communication failures and adverse events in hospitals. To address this, the I-PASS Handoff Bundle has been recently developed. I-PASS is a mnemonic: I for illness severity, $\mathrm{P}$ for patient summary, A for action list, $\mathrm{S}$ for situation awareness and contingency planning, $\mathrm{S}$ for synthesis by receiver (readback). Starmer et al ${ }^{16}$ described the details of I-PASS elsewhere.

In a paper published in 2014, Starmer et al ${ }^{17}$ described the process they used to develop, implement, and disseminate an innovative comprehensive handoff curriculum in pediatric residency training programs as a fundamental component of the multi-center Initiative for Innovation in Pediatric Education-Pediatric Research in Inpatient Settings Accelerating Safe Sign-outs (I-PASS) Study. They reported that $96 \%$ of residents and $97 \%$ of faculty agreed or strongly agreed that the curriculum promoted acquisition of relevant skills for patient care activities. They also shared lessons learned, including the importance of approaching curricular implementation as a transformational change effort, assembling a diverse team of junior and senior faculty to provide opportunities for mentoring and professional development, and linking the educational intervention with the direct measurement of patient outcomes. Although this curriculum was developed for pediatric residency programs, it might also be applicable to hospital respiratory care departments and respiratory care educational programs.

Starmer et $\mathrm{al}^{18}$ conducted an interventional study of resident handoffs in 10,740 admissions at 9 hospitals. They measured rates of medical errors, preventable adverse events, miscommunications, and resident workflow. The intervention was informed by I-PASS. Error rates were measured, and handoffs and workflow were assessed. There was a reduction in medical error rate by $23 \%$ and preventable adverse events by $30 \%$ after implementation of I-PASS. There were significant error reductions at 6 of the 9 participating sites and significant increases in the inclusion of all key elements. This occurred without significant changes in the time required for oral handoffs or in resident workflow. This provides strong support for the use of I-PASS, with impressive reductions in medical errors and preventable adverse events. What is most notable is that these results occurred despite $<60 \%$ post-implementation adherence with 4 of the 5 elements of I-PASS. The greatest adherence was for patient summary, but even that was $<80 \%$ post-implementation.

\section{Protocols and Multidisciplinary Teams}

Clinicians lacking expertise in the long-term management of tracheostomy often manage postoperative care following the tracheostomy procedure. Prolonged time to decannulation is associated with a high incidence of complications. To address this issue, Smith et a ${ }^{19}$ developed a low-risk tracheostomy clinical pathway. This decannulation pathway provided for an in-depth stepwise approach to initial cuff deflation, tracheostomy tube change, initiation of occlusion trials, and decannulation based on the patient meeting a series of defined milestones. Potentially eligible subjects underwent initial screening by their attending physician and/or a respiratory therapist (RT). After development of the pathway, a pilot group of 34 consecutive subjects underwent evaluation, of whom 13 were ineligible because of high-risk factors (upper-airway obstruction, unfavorable neck anatomy, or medical factors such as coagulopathy). A follow-up cohort of 107 consecutive subjects were studied to assess the sustainability of the pathway, and 39 met the low-risk criteria. Total time to decannulation in the baseline cohort was $15.5 \pm 12.1 \mathrm{~d}$. After implementation of the pathway in the pilot cohort, the time to decannulation decreased to $5.7 \pm 2.8 \mathrm{~d}$. In the follow-up cohort, time to decannulation was $8.1 \pm 7.1 \mathrm{~d}$. There was no association between adverse events and use of the pathway. The pathway was a safe and effective tool to guide clinicians in the management of tracheostomies. This pathway/protocol was largely implemented by RTs and illustrated a way that RTs can add value to the care of patients with tracheostomies.

In a quality improvement project, Gupta et $\mathrm{al}^{20}$ evaluated an RT-directed protocol in the setting of simple weaning (651 cases) and difficult weaning (131 cases). The RT protocol was successful in $79 \%$ with simple weaning and in $77 \%$ with difficult weaning. The RT protocol increased ventilator-free days by $21 \%$ with simple weaning and by $68 \%$ with difficult weaning. A multivariate analysis showed no significant difference between the RT-directed weaning and physician-directed weaning. This study adds to the accumulating evidence supporting the use of RT protocols for ventilator liberation.

In a prospective study, Klompas et $\mathrm{al}^{21}$ evaluated whether daily coordinated spontaneous awakening trials (SATs) 
and spontaneous breathing trials (SBTs) might reduce the likelihood of a ventilator-associated event (VAE). The study included 20 ICUs between November 2011 and May 2013. Twelve units $(5,164$ episodes of mechanical ventilation) implemented a collaborative protocol for nurses and RTs to perform daily paired SATs and SBTs, and 8 units conducted surveillance alone $(3,425$ episodes of mechanical ventilation). In the collaborative units, there were significant increases in SATs, SBTs, and percentage of SBTs performed without sedation. This was associated with significant decreases in duration of mechanical ventilation and hospital stay. Although there was no change in the number of VAEs per ventilator days, there was a significant decrease in the number of VAEs per episode of mechanical ventilation (OR of $0.63,95 \%$ CI $0.42-0.97$ ) and infection-related ventilator-associated complications (OR of $0.35,95 \%$ CI $0.17-0.71$ ), but not for pneumonia (OR of $0.51,95 \%$ CI $0.19-1.3)$. Within the surveillance-only units, there were no significant changes in SATs, SBTs, or VAEs. This study provides evidence to support not only the use of paired SAT/SBT, but also that this might positively affect VAEs, an indicator of quality for mechanically ventilated patients.

A multicomponent and multidisciplinary bundle of strategies for ventilator liberation and early mobilization has been proposed and is called the awakening and breathing coordination, delirium monitoring/management, and early exercise/mobility (ABCDE) bundle. Balas et $\mathrm{al}^{22}$ evaluated the effectiveness and safety of implementing the ABCDE bundle in everyday practice. This single-center study enrolled 146 subjects pre-bundle and 150 subjects post-bundle implementation. Subjects in the post-implementation period had 3 more ventilator-free days than did those in the pre-implementation period. Subjects managed with the ABCDE bundle had lower odds of delirium and increased odds of mobilizing out of bed at least once during an ICU stay. No significant differences were noted in self-extubation or re-intubation rates. Because the RT is responsible for implementing the SBTs, this is an example of how RTs can contribute to improved outcomes as part of a multidisciplinary team.

\section{Perioperative Obstructive Sleep Apnea}

OSA is prevalent among preoperative patients and has been associated with increased risk of postoperative complications. ${ }^{23}$ It has been suggested that as many as $90 \%$ of those with OSA are not yet diagnosed and therefore not treated. ${ }^{24}$ Mutter et al ${ }^{25}$ conducted a matched cohort analysis of polysomnography data and Manitoba health administrative data. Postoperative outcomes of subjects with OSA up to $5 \mathrm{y}$ before (undiagnosed OSA, $n=1,571$ ) and any time after (diagnosed OSA, $n=2,640$ ) polysomnography and prescription of CPAP for a new diagnosis of OSA were compared with controls with low risk of sleep apnea $(n=16,277)$. The risk of respiratory complications (OR of 2.08) similarly increased for both undiagnosed and diagnosed OSA. The risk of cardiovascular complications, primarily cardiac arrest and shock, was different between undiagnosed OSA (OR of 2.20) and diagnosed OSA (OR of 0.75). Important risk modifiers were OSA severity, type of surgery, age, and other comorbidities. The findings that diagnosis of OSA and prescription of CPAP were associated with a reduction in postoperative cardiovascular complications has important implications for respiratory care practice.

The STOP-BANG questionnaire is often used to estimate OSA risk, and the likelihood of having moderate or severe OSA increases with each point increase in the STOPBANG score. ${ }^{26}$ Subjects diagnosed with OSA are often treated with CPAP in the perioperative period. ${ }^{27}$ Proczko et $\mathrm{al}^{28}$ assessed postoperative hospital stay and pulmonary complications in 3 groups of morbidly obese subjects undergoing bariatric surgery. Group A comprised 99 subjects who were preoperatively diagnosed with OSA and who used CPAP before and after surgery. Group B consisted of 182 subjects who met at least 3 STOP-BANG criteria but were not diagnosed with OSA; these subjects did not use CPAP. Group C, the reference group, comprised 412 subjects who scored $<3$ items on the STOP-BANG questionnaire. In the perioperative period, Group B subjects had a significantly higher rate of pulmonary complications and increased hospital stay. There were also 2 cases of sudden death in this group.

\section{Readmissions}

Since the launch of the Hospital Readmission Reduction Program in 2013, hospitals in the United States with greaterthan-expected 30-d hospital readmission rates for acute myocardial infarction, pneumonia, congestive heart failure, COPD, and hip/knee arthroplasty are subject to penalties from the CMS (http://www.cms.gov/Medicare/ Medicare-Fee-for-Service-Payment/AcuteInpatientPPS/ Readmissions-Reduction-Program.html). The inclusion of pneumonia and COPD impact the practice of RTs. In 2014, Feemster and $\mathrm{Au}^{29}$ critically assessed the use of all-cause 30-d COPD readmissions as an accountability measure, discussing benefits and highlighting the substantial drawbacks and unintended consequences of the measure that could adversely affect providers, hospitals, and patients with COPD.

Prescott et $\mathrm{al}^{30}$ reviewed 12 studies related to readmission after pneumonia hospitalization. All-cause 30-d readmission rates ranged from 16.8 to $20.1 \%$, with $15.6 \%$ in studies from the United States. Pneumonia, cardiovascular disease (including heart failure), and COPD are the most common reasons for early readmission after pneumonia 
discharge. Although it was the most common readmission diagnosis, pneumonia accounted for a minority of all readmissions. The authors correctly suggest that further research is needed to understand how hospitalization for pneumonia fits within the broader context of the health trajectory of patients.

Due to the poor discriminative ability of existing models to predict 30-d readmission for pneumonia, Mather et al ${ }^{31}$ probed the potential for additional predictive variables. The 30-d readmission rate was $15.5 \%$ of the 965 subjects. The variables significantly associated with allcause $30-\mathrm{d}$ readmissions were male (OR 1.59), 3 or more previous admissions (OR 1.84), chronic lung disease (OR 1.63), cancer (OR 2.18), income $<\$ 43,000$ (OR 1.82), history of anxiety or depression (OR 1.62), and hematocrit $<30 \%$ (OR 1.86). Compared with the model using only the CMS variables, the addition of socioeconomic status and health-care utilization variables significantly improved model performance.

Using a large national commercial insurance database, Sharif et al ${ }^{32}$ examined $30-\mathrm{d}$ readmission in 8,263 subjects with COPD. The $30-\mathrm{d}$ readmission rate was $8.9 \%$. Patient factors, provider factors, and system factors were associated with 30-d readmission. These results suggest that one of 11 subjects hospitalized with COPD is readmitted within $30 \mathrm{~d}$ of discharge, with many important and modifiable risk factors for early readmission.

Werre et $\mathrm{al}^{33}$ conducted a retrospective study comparing the effects of therapist-directed (protocol) and physiciandirected (non-protocol) respiratory therapy on hospital stay and 30-d readmission in subjects with COPD and acute bacterial pneumonia. There was no significant interaction between protocol use, age, and disease severity on hospital stay. Disease severity had a significant effect on hospital stay. The 30-d readmission rate was lower with the use of the protocol. The finding of no difference in hospital stay with the use of the RT protocol indicates that it did not sacrifice treatment efficacy. More important, the RT-directed protocol conferred benefit by reducing $30-\mathrm{d}$ readmission. This study provides additional evidence supporting the use of RT protocols.

\section{Summary}

There are a number of areas in which patient safety impacts respiratory care practice. This paper focused on studies published in 2014 related to pressure injury, handoffs, protocols and multidisciplinary teams, perioperative OSA, and readmissions.

\section{REFERENCES}

1. Kohn LT, Corrigan JM, Donaldson MS. To err is human: building a safer health system. Washington, DC: National Academies Press; 2000 .
2. Committee on Quality of Health Care in America and Institute of Medicine. Crossing the quality chasm: a new health system for the 21st century. Washington DC: National Academies Press; 2001.

3. Davies HT, Nutley SM, Mannion R. Organisational culture and quality of health care. Qual Health Care 2000;9(2):111-119.

4. Nieva VF, Sorra J. Safety culture assessment: a tool for improving patient safety in healthcare organizations. Qual Saf Health Care 2003; 12(Suppl 2):ii17-ii23.

5. Morello RT, Lowthian JA, Barker AL, McGinnes R, Dunt D, Brand C. Strategies for improving patient safety culture in hospitals: a systematic review. BMJ Qual Saf 2013;22(1):11-18.

6. Dicuccio MH. The relationship between patient safety culture and patient outcomes: a systematic review. J Patient Saf 2014 [Epub ahead of print].

7. Rosenthal MB. Nonpayment for performance? Medicare's new reimbursement rule. N Engl J Med 2007;357(16):1573-1575.

8. Hess DR. The evidence is in: noninvasive ventilation saves lives. Crit Care Med 2015;43(4):927-928.

9. Gay PC. Complications of noninvasive ventilation in acute care. Respir Care 2009;54(2):246-257; discussion 257-248.

10. Nava S, Navalesi P, Gregoretti C. Interfaces and humidification for noninvasive mechanical ventilation. Respir Care 2009;54(1):71-84.

11. Barros LS, Talaia P, Drummond M, Natal-Jorge R. Facial pressure zones of an oronasal interface for noninvasive ventilation: a computer model analysis. J Bras Pneumol 2014;40(6):652-657.

12. Yamaguti WP, Moderno EV, Yamashita SY, Gomes TG, Maida AL, Kondo CS, et al. Treatment-related risk factors for development of skin breakdown in subjects with acute respiratory failure undergoing noninvasive ventilation or CPAP. Respir Care 2014;59(10):15301536.

13. Fisher DF, Chenelle CT, Marchese AD, Kratohvil JP, Kacmarek RM. Comparison of commercial and noncommercial endotracheal tube-securing devices. Respir Care 2014;59(9):1315-1323.

14. Wagner JL, Shandas R, Lanning CJ. Extubation force depends upon angle of force application and fixation technique: a study of 7 methods. BMC Anesthesiol 2014;14:74.

15. Hartzell LD, Havens TN, Odom BH, Stillman TG, Boswell JL, Bower CM, Richter GT. Enhanced tracheostomy wound healing using maltodextrin and silver alginate compounds in pediatrics: a pilot study. Respir Care 2014;59(12):1857-1862.

16. Starmer AJ, Spector ND, Srivastava R, Allen AD, Landrigan CP, Sectish TC, I-PASS Study Group. I-PASS, a mnemonic to standardize verbal handoffs. Pediatrics 2012;129(2):201-204.

17. Starmer AJ, O'Toole JK, Rosenbluth G, Calaman S, Balmer D, West DC, et al. Development, implementation, and dissemination of the I-PASS handoff curriculum: a multisite educational intervention to improve patient handoffs. Acad Med 2014;89(6):876-884.

18. Starmer AJ, Spector ND, Srivastava R, West DC, Rosenbluth G, Allen AD, et al. Changes in medical errors after implementation of a handoff program. N Engl J Med 2014;371(19):1803-1812.

19. Smith KA, Matthews TW, Dubé M, Spence G, Dort JC. Changing practice and improving care using a low-risk tracheotomy clinical pathway. JAMA Otolaryngol Head Neck Surg 2014;140(7):630-634.

20. Gupta P, Giehler K, Walters RW, Meyerink K, Modrykamien AM. The effect of a mechanical ventilation discontinuation protocol in patients with simple and difficult weaning: impact on clinical outcomes. Respir Care 2014;59(2):170-177.

21. Klompas M, Anderson D, Trick W, Babcock H, Kerlin MP, Li L, et al. The preventability of ventilator-associated events. The CDC Prevention Epicenters Wake Up and Breathe Collaborative. Am J Respir Crit Care Med 2015;191(3):292-301.

22. Balas MC, Vasilevskis EE, Olsen KM, Schmid KK, Shostrom V, Cohen MZ, et al. Effectiveness and safety of the awakening and 


\section{Year In Review 2014: Patient SAfety}

breathing coordination, delirium monitoring/management, and early exercise/mobility bundle. Crit Care Med 2014;42(5):1024-1036.

23. Chung SA, Yuan H, Chung F. A systemic review of obstructive sleep apnea and its implications for anesthesiologists. Anesth Analg 2008; 107(5):1543-1563.

24. Finkel KJ, Searleman AC, Tymkew H, Tanaka CY, Saager L, SaferZadeh E, et al. Prevalence of undiagnosed obstructive sleep apnea among adult surgical patients in an academic medical center. Sleep Med 2009;10(7):753-758.

25. Mutter TC, Chateau D, Moffatt M, Ramsey C, Roos LL, Kryger M. A matched cohort study of postoperative outcomes in obstructive sleep apnea: could preoperative diagnosis and treatment prevent complications? Anesthesiology 2014;121(4):707-718.

26. Chung F, Yang Y, Liao P. Predictive performance of the STOP-Bang score for identifying obstructive sleep apnea in obese patients. Obes Surg 2013;23(12):2050-2057.

27. Bakker JP, Balachandran JS, Tecilazich F, Deyoung PN, Smales E, Veves A, Malhotra A. Pilot study of the effects of bariatric surgery and continuous positive airway pressure treatment on vascular function in obese subjects with obstructive sleep apnoea. Intern Med J 2013;43(9):993-998.
28. Proczko MA, Stepaniak PS, de Quelerij M, van der Lely FH, Smulders JF, Kaska L, Soliman Hamad MA. STOP-Bang and the effect on patient outcome and length of hospital stay when patients are not using continuous positive airway pressure. J Anesth 2014;28(6):891897.

29. Feemster LC, Au DH. Penalizing hospitals for chronic obstructive pulmonary disease readmissions. Am J Respir Crit Care Med 2014; 189(6):634-639.

30. Prescott HC, Sjoding MW, Iwashyna TJ. Diagnoses of early and late readmissions after hospitalization for pneumonia. A systematic review. Ann Am Thorac Soc 2014;11(7):1091-1100.

31. Mather JF, Fortunato GJ, Ash JL, Davis MJ, Kumar A. Prediction of pneumonia 30-day readmissions: a single-center attempt to increase model performance. Respir Care 2014;59(2):199-208.

32. Sharif R, Parekh TM, Pierson KS, Kuo YF, Sharma G. Predictors of early readmission among patients 40 to 64 years of age hospitalized for chronic obstructive pulmonary disease. Ann Am Thorac Soc 2014;11(5):685-694

33. Werre ND, Boucher EL, Beachey WD. Comparison of therapistdirected and physician-directed respiratory care in COPD subjects with acute pneumonia. Respir Care 2015;60(2):151-154. 